\title{
On the Structural Complexity of Natural Language Sentences
}

\author{
Dekang Lin* \\ Artificial Intelligence Laboratory \\ Massachuset ts Institute of 'l'echuology \\ Rm 767, 545 'Technology Square \\ Cambridge, Massachusetts, USA, 02139 \\ E-mail: lindek@ai.mit.edu
}

\begin{abstract}
T'he objective of this paper is to fomalize the intuition about the complexity of syntactic structures. We propose a definition of structural complexity such that sentences ranked by our definition as nore complex are generally nore difficult for humans to process. We justify the definition by showing how it is able to account for several seemingly unrelated phenomena in natural languages.
\end{abstract}

\section{Introduction}

Intuitively, certain syntactic structures are more diflicult for humans to process than others. For example, compare the following to sentences:
a. The cat that the dog that the man bought chased died.
b. 'The man bought the dog that chased the cat that died.

It is obvious that sentence ( 1 a) is much more difficult to understand than (1b). Since the two sentences are of the same length and involve the same set of semantic relationships, the difficulty in understanding (la) can only be attributed to its syntactic structure.

'The objective of this paper is to formalize the intuition about the complexity of syutactic structures. We propose a definition of structural complexity (SC) such that sentences ranked by our definition as more complex are generally more difficult for humans to process than otherwise similar sentences. In other words, suppose a pair of sentences $A$ and $B$ consist of the same set of words and have essentially the same meaning, then sentence $\Lambda$ is more difficult to process than sentence $\mathrm{B}$ if $\mathrm{SC}(\mathrm{A})>\mathrm{SC}(\mathrm{B})$. For example, the proposed definition of structural complexity correctly pre-

\footnotetext{
*On leave from the University of Manitoba, Winnipeg, Manitoba, Canada. 'This research has been supported by NSERC: Rescarch Grant OCP121338. 'Tho author is very grateful to the reviewers who pointed ont several mistakes in the draft.
}

dicts that ( 1 a) is much more difficult to process than (lb).

the notion of structural complexity proposed in this paper offers explanations for a set of secmingly unrelated phenomena:

- We will show that the definition of structulal complexity explains why a Intch sentence involving cross-serial dependencies is slightly easier to understand than a corresponding conter-embedded German sentence.

- We will also show that extrapositions, such as heavy-NP shift and PP' extractions are motivated by reducing syntactic complexity. The extraposition of an element is only warranted when the structural complexity of the sentence is reduced as a result.

- NP modifiers of a head tend to be closer to the head than its PP modifiers, which in tum tend to be closer than its CP (clausal) modifiers. In Generalized Phrase Strcuture Grammar (GPSG) (Gazdar ot al., 1985), these linear order constraints are stated explicitly in the grammar. The notion of structural complexity provides an explanatory accomnt.

'lhere are several reasons why the notion of structural complexity is useful. Firstly, in natural language generation, a generator should generate the simplest sentence that conveys the intended meanings. Structural complexity can be used to choose the syntactic structures with the lowest structural complexity so that the resulting sentence is casier to understand than other alternatives.

Secondly, structural complexity is also needed in assessing the readability of documents. It is well known that the length of a sentence is not a reliable indicator of its readability. Yet, the readability of texts has up to now been measured by the lengths of sentences and familiarities of the words in the documents. Using structural complexity instead of sentence length allows the readability of clocuments to be measured more accurately.

Finally, we propose, in Section 4, that extrapositions are motivated by reduction of structural 
complexity. In other words, extrapositions are only allowed if the structural complexity of the sentence is reduced as a result. This constraint is useful both in parsing sentences with extrapositions and in deciding where to use extraposition during generation.

The notion of structural complexity is defined in Scction 2. We then justify the definition of structural complexity by demonstrating in Sections 3, 4 , and 5 that sentences with lower structural complexity are easier to understand than otherwise similar sentences with higher structural complexity.

\section{Structural Complexity}

The definition of structural complexity presumes the notion of dependency relationships between words in a sentence. In dependency grammars (Hudson, 1984; Mel'čuk, 1987), a dependency relationship is a primitive relationship between two words, called the head and the modifier. In constituency grammars that contain the X-bar theory as a component, dependency relationships between words are implicitly specified in X-bar structures. The modifiers of a word $w$ are the head words of the specifier, complements, and adjuncts of $w$. For example, Figure 1 is the X-bar structure of (2). 'The word "will" has two modifiers: the head word of its NP specifier ("Kim") and the head word of its VP complement ("bring"). 'Ihe dependency relationships in the X-bar structure in Figure 1 are shown in Figure 2. Each directed link in rigure 2 represents a dependency relationship with the direction going from the head to the modificr.

(2) Kim will bring the wine in the evening.

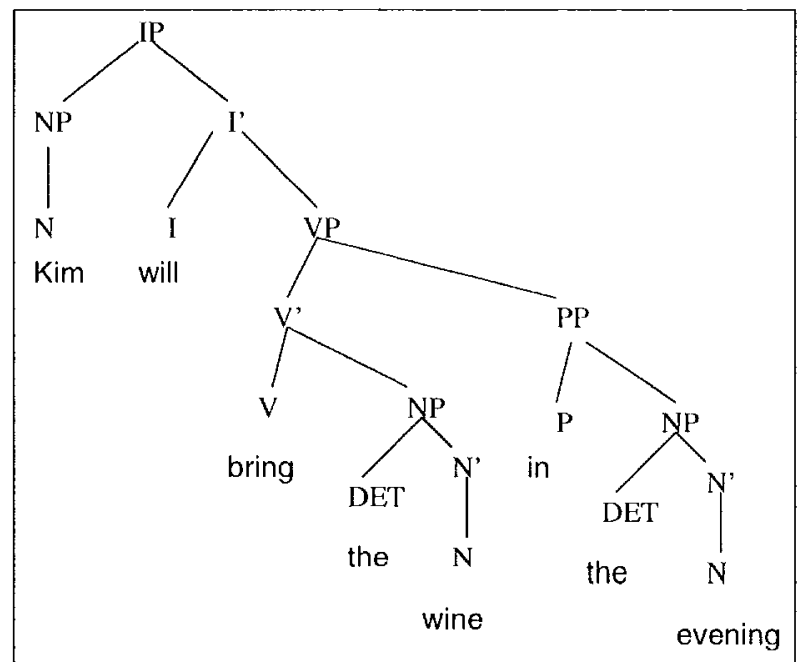

Figure 1: X-bar structures of (2)

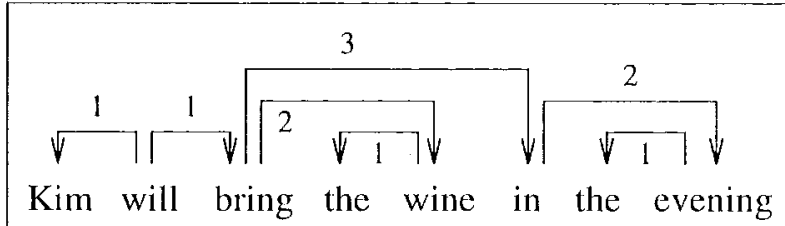

ligure 2: Dependency structure of (2)

In order to recognize the structure of a sentence, a parser must establish the dependency links botween the words in the sentence. Structural complexity measures how easy or difficult it is to ostablish these dependency links. 'The definition of structural complexity is based on the assumption that the shorter dependency links are easier to establish than longer ones, where the length of a dependency link is one plus the number of words between the head and the modifier. For example, the lengths of the links in rigure 2 are shown by the numbers attached to the dependency links.

\section{Definition 2.1 (Structural Complexity)}

The structural complexity of a dependency structure is the total length of the dependency links in the structure.

For example the structural complexity of the dependency structure in Figure 2 is 11 .

In the next three sections, we will show that the definition of structural complexity does indeed reflect the difficulty in processing a sentence. We will present examples in which sentences with lower structural complexitics are easier to process than similar sentences with higher structural complexitios.

\section{Center embedding}

'The difficulty in processing center cmbedding sontences, such as (1a), has been explained by its requirement on the size of the stack in a parser. This explanation presumes that the human parser uses a push-down stack to store the partially built, constituents. 'I'he notion of structural complexity provides an explanation of the difficulty of processing center embedding that makes much weaker commitment to the parsing model. ligure 3 shows the lengths of the dependency links in a center-embedding sentence (1a) and a noncenter-embedding sentence (1b) with similar semantics. The structural complexity of the centorembedding sentence is 30 , which is much higher than the structural complexity $(=1.2)$ of the noncenter-cmbedding sentence.

The presumption that human sentence processor uses a push-down stack is challenged by the contrast between cross-scrial dependencies in Dutch (e.g., Figure 4a) and center-embedding scntences in German (e.g., Figure 4b.)

Since the cross serial dependencies are much more difficult to handle with push-down stacks 


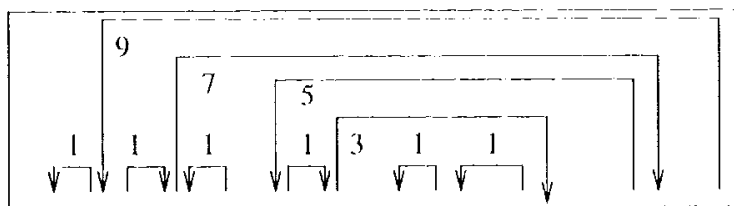

The cat that the dog that the man bought chased died

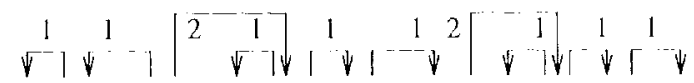

The man bought the dog that chased the cat that died

ligure 3: Conter-limbedding vs. Non-Centertimbedding Sentences

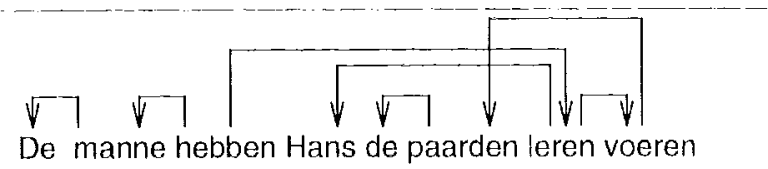

The man have Hans the horses teach feed

a. Dutch: cross serial dependency, structural complexity $=13$

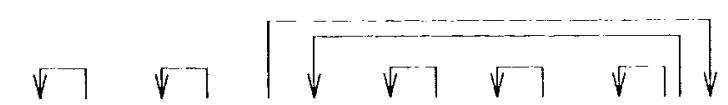

Die Maenner haben Hans die Pferde fuettern gelehrt

The men have Hans the horsesfeed teach

b. Genman: center cmbedding, structural complexity $=14$

\section{$\sqrt{ }|\sqrt{ }| \sqrt{ } \sqrt{ } \sqrt{\sqrt{1}} \downarrow$}

The men taught Hans to feed the horses

c. English: right branching, structural complexity $=$ )

ligure 1: cross-serial dependency vs. conterembedeliug vs. right-loranching

than nested dependencies, the hypothesis that human parser uses a push-down stack would predict that the Dutch sentences such as Figure $4 a$ should be much more diflicult to understand than the corresponding German sentences with nested dependencies (Figure 4b). However, data from psycho-linguistic experiments suggest that they are in fact slightly casier to process than the corresponding German sentences with nested dependencies (Bach et al., 1986). 'T'his observation can be accounted for by structural complexity, since the structural complexity of the 1)utch sentence (Figure 4a) is 13 , which is slightly lower than the structural complexity $(=14)$ of the corresponding (German senlence tigure 4l). It was also observed in (13ach et al., 1986) that "l'or someone with even a limited competence in Finglish and either of the other languages, the patterns in Dutch and (rerman seem to be more difficult to process and pro- duce than their finglish comberpates" (1). 249). "This is also consistent with the structural conl" plexity account, since the structural complexity of Figure 4c is 9, which is significantly lower than its Dutich and Goman comberparts (Figure ha and 4b).

\section{Extrapositions}

Extraposition refors to the movenent of an clement from its nomal position lo a position at or near the ond of the sentence. lixamples of extra. position in ringlish inclucle:

\section{Heavy-NP'shifi:}

(3) a. Joo sent the book he found in Paris to his pal

b. Joe sont to his pal the book he fonnd in Paris

\section{Fxtraposed rolative}

(4) a. A man that no one knew stood up

b. A man stood up that no one knew

\section{PP-cxtraposition}

(5) a. I read a description of llockney's latest picture yesterday

b. I read a description yesterday of llockney's latiest picture?

\section{Extraction from AP}

(6) a. How certain that the Mets will win are you?

b. Ilow cortain are you that he Mets will win?

Mechanism for constraining extaposition is $11 \mathrm{r}$ gentily needed in both parsing and generation. 'T'o the best of the author's knowledge, none of the broad-coverage parsers or gemerators handles extrapositions in a principled fashion. The reason for this is that extrapositions appear to be dependent upon certain aspects of contexts that are not captured by usual syntactic features. For example, compare the following pair of sentences

a. I talked with a man yeslerday with a mustache

b. * l talked with a man one ycar and four monlhs ago with a mustiache

The synlactic structures of (7a) and (7b) are the sane, which is shown in Figure 5, except that the adverbial phrase $\Lambda d v l$ ' is "yesterday" in (7a) and "one year and four months ago" in (7b). Although the two adverbial phrases are two diflerent strings, they are identical in their syntactic foatures. Yet, extraposition is good in (7a) but bad in (7b).

Wo propose that the purpose of extraposition is to make a sentence casior to understand. 'Therefore, cxtraposition is only allowed when the stinctural complexity of the sentence is redued as a result. Note that reduction of structural complexity is not the only constraint on extraposition. 'There 


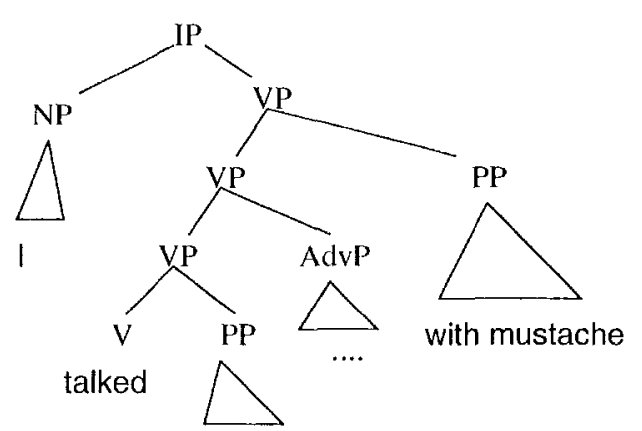

to a man

Figure 5: Parse tree of (7a) and (7b)

are also syntactic constrains such as Right Roof Condition (Ross, 1967) or Complement Principle (Rochemont and Culicover, 1990).

When a phrase is extraposed, the set of dependency relationships rcmains the same. However, the lengths of some of the dependency links will change. 'The structural complexity of the sentence may change as a result. Figure 6 illustrates how cxtrapositions affect the lengths of dependency links in (3), (4), (5), and (6). Only the dependency links whose lengths arc changed are shown there. In all cases, structural complexity is reduced by the extraposition.

Consider the difference between (7a) and (7b). In (7a), the extraposition of [PP with a mustache] increases the length of the dependency link between "man" and "with" by 1, but reduces the length of the dependency between "talked" and "yesterday" by 3. 'Therefore, the structural complexity is reduced by 2 as a result of the extraposition. In contrast, in (7b), the extraposition of [PP with a mustache] increases the length of the dependency link between "man" and "with" by 6 and roduces the length of the dependency link between "talk" and "ago" by 3 . Thus the structural complexity is increased when [PP with a mustache] is extraposed.

The hypothesis that extraposition must reduce the structural complexity also explains why in heavy-NP shift, the extraposed NP must be heavy, i.e., consisting of many words. When the complement NP' of a verb is 'shifted' to the right across an adjunct modifier of the verb, the length of the dependency link from the verb to the head of the NP is increased by length the adjunct modifier. On the other hand, the length of the dependency link from the verb to the adjunct modifier is reduced by the length of the NP. Therefore, the structural complexity of the sentence can only be reduced as a result of the extraposition when the $\mathrm{NP}$ is longer than the adjunct modifier,

Joe sent to his pal the book he found in Paris
(a) Heavy-NP shift, SC reduction $=(7+2)-(5+1)=3$
A man that no one knew stood up
A man stood up that no one knew
(b) Extraposed relative clausc, SC reduction=(5+1)-(3+1)=2
I read a description of Hockney's latest picture yesterday
I read a description yesterday of Hockney's latest picture
(c) PP-extraposition, SC reduction=(7+1)-(4+2)=2
Figure 6 : Extraposition must reduce structural
complexity
How certain are you that the Mets will win
(d) Extraction from AP, SC reduction=(6+1)-(3+1)=3
How certain that the Mets will win are you
I

\section{$5 \quad$ Linear Precedence}

In most languages, the NP modifiers of a word tend to be closer to the word than its PP modifiers, which, in turn, tend to be closer to the word than its Cl' (clausal) modifiers. In GPSG (Gazdar et al., 1985), these linear order constraints are stated explicitly as the linear precedence rules. In this section, we show that the linear precedence rules in GPSG can be derived from the assumption that the linear order among different types of modifying phrases, such as NP, PP, and CP, should minimize the structural complexity so that the sentence is as easy to process as possible.

Suppose a word $w$ has $n$ modifiers $\mathrm{XP}_{1}, \mathrm{XP}_{2}$, $\ldots, \mathrm{XP}_{n}$; the number of words in $\mathrm{XP}_{i}$ is $l_{i}$; and the head word of $\mathrm{XP}_{i}$ is $\mathrm{w}_{i}$, which is the $p_{i}$ 'th word in $\mathrm{XP}_{i}$. Without loss of generality, lct us assume that $w$ precedes its modifiers. If the order of the modifiers is $\mathrm{XP}_{1}, \mathrm{XP}_{2}, \ldots, \mathrm{XP}_{n}$, then the length of the dependency link between $w$ and the head of $\mathrm{XP}_{i}$ is $\left(p_{i}+\sum_{j=1}^{i-1} l_{j}\right)$ and the total length of dependency links within the maximal projection 
of $\mathrm{w}$ is:

$$
\begin{aligned}
& \sum_{i=1}^{n}\left(p_{i}+\sum_{j=1}^{i-1} l_{j}\right) \\
& =(n-1) l_{1}+(n-2) l_{2}+\ldots+l_{n-1}+\sum_{i=1}^{n} p_{i}
\end{aligned}
$$

Among all permutations of $\mathrm{XP}_{1}, \mathrm{XP}_{2}, \ldots, \mathrm{XP}_{n}$, the above sum is the minimal when $l_{1} \leq l_{2} \leq \ldots \leq$ $l_{n}$. In other words, the total length of dependency links is minimal when the modifiers with fewer words are closer to the head. Generally speaking, P'Ps contain more words than Nl's and CP's contain more words than PPs. 'T'herefore, NP modifiers should be closer to the head word than P' modifiers and PP modifiers should be closer to the head word than CI' modiliors if the structural complexity of the maximal projection of the head word $w$ is to be minimized.

\section{Discussion}

We used the total length of the dependency links in the definition of structural complexity. 'The examples presented in the previous sections are also consistent with a definition that uses the maximum length of structural links. 'The reason we choose to use the sum is that the definition naturally incorporate the length into consideration.

The arguments presented in previous sections are preliminary. Our future work include backing up the hypothesis with empirical evidence and investigate the application of structural complexity in handling extraposition in parsing and generation.

\section{Conclusion}

We have proposed a notion of structural complexity. A sentence with higher structural complexity is more difficult to process than a similar sentences with lower structural complexity. Structural complexity is needed in both parsing and generation. It can also be used to assoss the readability of documents. Wo support the definition of structural complexity with a set of secmingly unrelated phenomena: the contrast between centerembedding and right-branching sentences, cxtrapositions, and the linear order among modifying phrases. In all of these cases, sentences with lower structural complexity are casier to understand.

\section{References}

F. Bach, C, Brown, and W. Marslen-Wilson. 1986. Crossed and nested dependencies in German and Dutch: $\Lambda$ psycholinguistic study. Language and Cognitive Processes, I(4):24.9 262.

Gerald Gazdar, Fwan Klein, Geoflery Pullum, and Ivan Sag. 1985. Generalized Phrase Structure Grammar. Basil Blackwell Publisher Ltd, Oxford, UK.
Richard Hudson. 1984. Word Grammar. Basil Blackwell Publishers limited., Oxford, England.

lgor A. Mol'čuk. 1987. Dependency syntax: theory and practice. State University of New York Press, Albany.

Michad S. Rochemont and Peter W. Culicover. 1990. linglish Hocus Constructions and the Theory of Crammar. Cambridgo Studies in Linguistics. Cambridge University Pross.

J. Ross. 1967. Constraints on variables in synlax. I'h.I). Hhesis, M.I.'I', Cambridge, MA. 